\title{
Davunetide improves spatial learning and memory in Alzheimer's disease-associated rats
}

\author{
Jun Zhang a, Shu-Yu Wei ${ }^{\text {b }}$, Li Yuan ${ }^{\text {a }}$, Lin-Lin Kong a , Sheng-Xiao Zhang a , Zhao-Jun Wang a , \\ Mei-Na Wu ${ }^{a}$, Jin-Shun $\mathrm{Qi}^{\mathrm{a}, *}$ \\ a Department of Physiology, Shanxi Medical University, Taiyuan 030001, PR China \\ b Department of Severe Liver Disease, The 3ird People's Hospital of Taiyuan, Taiyuan 030012, PR China
}

\section{H I G H L I G H T S}

- Davunetide protected against A $\beta-42$ induced impairment of spatial learning and memory in rats

- Davunetide reversed $A \beta$-induced suppression of hippocampal LTP in rats

- Davunetide mostly restored the level of phospharylated AKT in rat hippocampus

\section{A R T I C L E I N F O}

\section{Article history:}

Received 28 December 2016

Received in revised form 25 February 2017

Accepted 27 February 2017

Available online 28 February 2017

\section{Keywords:}

Davunetide

Amyloid $\beta$ protein

Spatial memory

Long term potentiation

Phosphorylated AKT

Synaptic plasticity

\begin{abstract}
A B S T R A C T
Memory loss and cognition decline are the main clinical manifestations of Alzheimer's disease (AD). Amyloid $\beta$ protein $(A B)$ aggregated in the brain is one of the key pathological characteristics of $A D$ and responsible for the deficits in learning and memory. It is reported that davunetide, an octapeptide derived from activitydependent neuroprotective protein (ADNP), inhibited $A \beta$ aggregation and $A \beta$-induced neurotoxicity. To further characterize the neuroprotective roles of davunetide and its possible mechanism, the present study investigated the effects of davunetide on Aß1-42-induced impairments in spatial memory, synaptic plasticity and hippocampal AKT level. In Morris water maze (MWM) test, bilateral intrahippocampal injection of A $31-42$ significantly increased escape latency and decreased target quadrant swimming time of rats, while three weeks of intranasal application of davunetide reversed the AB1-42-induced learning deficits and memory loss in a dosedependent manner. In vivo field potentiation recording showed that $A \beta 1-42$ suppressed long-term potentiation (LTP) of excitatory postsynaptic potential (fEPSP) in the hippocampal CA1 region of rats, while davunetide effectively blocked the suppression of LTP, without affecting paired-pulse facilitation (PPF). Western blotting experiments showed a significant decrease in the level of hippocampal p-AKT (Ser473), not total AKT, in AB1-42 only group, which was mostly antagonized by davunetide treatment. These findings demonstrate that davunetide, probably by enhancing PI3K/AKT pathway, plays an important positive role in attenuating AB1-42-induced impairments in spatial memory and synaptic plasticity, suggesting that davunetide could be an effective therapeutic candidate for the prevention and treatment of neurodegenerative disease such as AD.
\end{abstract}

(C) 2017 Elsevier Inc. All rights reserved.

\section{Introduction}

The main manifestation of Alzheimer's disease (AD) in the early stage is gradual deficits in the cognitive function. With the development of the disease, spatial disorientation, loss of motivation, abnormal behaviors, and serious dementia gradually appeared [1]. One of the pathological hallmarks of AD is high density of senile plaques (SPs), which

\footnotetext{
* Corresponding author at: Department of Physiology, Shanxi Medical University, 86 Xinjian South Road, Taiyuan, Shanxi 030001, PR China.

E-mail address: jinshunqi@sxmu.edu.cn (J.-S. Qi).
}

aggregated in the brain especially in the hippocampus and cerebral cortex. The main component of SP is amyloid $\beta$ protein $(A \beta)$ derived from amyloid precursor protein (APP) [2]. In two forms of natural $A \beta$, i.e. $A \beta 1-40$ and $A \beta 1-42$ [3], $A \beta 1-42$ is easier to aggregate early, and has more neurotoxicity than $A \beta 1-40$ [4]. Moreover, $A \beta$ oligomers rather than $A \beta$ fibrils have more neurocytotoxicity $[5,6]$. Many studies have confirmed that intracerebral injection of $A \beta 1-42$ could damage spatial memory and synaptic transmission of animals $[7,8]$. Our previous studies also found that $A \beta 1-42$ injection inhibited hippocampal long term potentiation (LTP) in rat $[9,10]$. However, the molecular mechanisms underlying $A \beta$ toxicity remain to be clarified and the effective neuroprotectants against $A \beta$ are urgently needed to be explored. 
Activity-dependent neuroprotective protein (ADNP) was originally isolated from conditioned medium of astrocytes treated with vasoactive intestinal peptide (VIP). Thereafter, ADNP has been shown to have potent neuroprotective actions among the neurotrophic factors secreted by glial cells [11]. In human and mouse brains, ADNP is predominantly expressed in the cerebellum, hippocampus and cerebral cortex [12]. Moreover, the expression of ADNP in the brain has been related to not only the neurogenesis in the hippocampus but also the early or mild AD progression. For example, ADNP expression was higher in 6-month-old PS1XAPP mice hippocampus than in WT mice [13]. A most recent study showed a significant association between greater cortical $A B$ and lower ADNP mRNAs. This supported that ADNP is essential for brain formation and linked to cognitive functions [14]. Davunetide (also namely NAP), an eight amino acid (NAPVSIPQ) neuroprotective peptide, is derived from ADNP, which has similar structural and functional characteristics to the active core of ADNP. It has been confirmed that davunetide has potent biological activities. Low concentration davunetide protected astrocytes against zinc toxicity [15], promoted neurite outgrowth in PC-12 cells and hippocampal neurons and increased synaptophysin expression in both rat hippocampal and cortical cultures [16,17]. A most recent article reported that ADNP/NAP dramatically increased microtubule end-binding protein-Tau interaction, suggesting a novel target for endogenous ADNP neuroprotection [18]. Also, davunetide prevented aggregation of $A \beta$ peptide in vitro [19] and $A \beta$-induced cellular toxicity in rat cerebral cortex neurons $[16,17]$. It seems that davunetide has beneficial effects on the neurodegenerative disorders such as AD.

However, the in vivo neuroprotective effects of davunetide against neurotoxic $A \beta$ and its molecular mechanism remain largely unknown. The present study, therefore, first investigated the protective effects of chronic administration of davunetide on AB1-42induced impairments in spatial learning and memory of rats by using Morris water maze (MWM) tests. Then, we explored the probable electrophysiological and molecular mechanisms by recording in vivo hippocampal long-term potentiation (LTP) and examining PI3K/AKT signal pathway in the hippocampus with field potential and western blotting techniques.

\section{Materials and methods}

\subsection{Animals and drugs}

All experiments were conducted on male Sprague-Dawley (SD) rats aged between 3 and 4 months. The rats, provided by the Beijing Experimental Animal Center of Military Medical Sciences, were housed in cages with ad libitum access to food and water, $12 \mathrm{~h}$ light/dark cycle and $20-25{ }^{\circ} \mathrm{C}$ temperature. All animal procedures were conducted in accordance with the Guidelines for Care and Use of Laboratory Animals and were approved by the Institutional Animal Research Ethics Committee at Shannxi Medical University. Amyloid $\beta$ peptide (Aß1-42) was ordered from Abcam (UK). Davunetide was synthesized by the Shanghai GL Biochem Ltd. (Shanghai, China) with a purity of $99.37 \%$ detected by reversed-phase high performance liquid chromatography (RP-HPLC). AB1-42 oligomer solution was prepared by the following procedures: dissolving $A \beta 1-42$ in $100 \%$ HFIP at a concentration of $1 \mathrm{mg} / \mathrm{ml}$; incubating the solution at $20-25{ }^{\circ} \mathrm{C}$ for $1 \mathrm{~h}$ under vortexing at a medium speed; sonicating the solution in a water bath sonicator for $10 \mathrm{~min}$; after dividing into small volume and drying with nitrogen gas, quantitative $A \beta 1$ 42 was stored at $-80{ }^{\circ} \mathrm{C}[9,20]$. DMSO was used to resuspend the A 1-42 before using. After D-PBS was added for a working solution $2.5 \mu \mathrm{g} / \mu \mathrm{l}$, the solution was incubated with occasional vortexing at a medium speed and left at $4{ }^{\circ} \mathrm{C}$ for $24 \mathrm{~h}$ to allow for peptide aggregation into oligomers [21].

\subsection{Surgical and drug administration}

After one week of acclimation, SD rats were randomly divided into four groups ( $n=10$ for each group): veilcle + saline, veilcle $+A \beta 1$ 42, Davunetide + saline, and Davunetide $+A \beta 1-42$. The protocol of bilateral hippocampal injection was previously described [22,23]. The animal was anesthetized using 5\% chloral hydrate and fixed on a digital display stereotaxic instrument (RWD Life Science, China). $2 \mu \mathrm{l}$ A $1-42$ solution (or vehicle) was injected into the bilateral hippocampus of rats by micro-injection pump (KD Scientific, USA) connecting a quartz capillary injection tube. The coordinates of injection tube tip were: anterior-posterior, $-3.0 \mathrm{~mm}$; medial-lateral, $-2.2 \mathrm{~mm}$; dorsoventral, $-3.0 \mathrm{~mm}$ to the bregma as a reference point. Intranasal administration of davunetide: from the next day after surgical, animals received a daily intranasal injection of davunetide ( $2.5 \mu \mathrm{g}$ in $10 \mu \mathrm{l}$, half in each nostril) or saline $(10 \mu \mathrm{l})$ [24,25] for 3 weeks. During behavioral tests, daily davunetide administration was still carried on.

\subsection{Morris water maze test}

Classical Morris water maze (MWM) test was used to detect animal's ability of long term spatial memory. The water maze apparatus is a circular stainless steel basin (150 cm in diameter and $60 \mathrm{~cm}$ deep) with different cues on the inner surface painted black. The maze was filled with water at a temperature of $22{ }^{\circ} \mathrm{C}$ [26]. A CCD camera was installed right above the sink to record the animal's swimming track. Behavior software (Ethovision 3.0, Noldus Information Technology, Netherlands) was used to analyze swimming distance and time of animals. Hidden platform acquisition was performed firstly in consecutive five days to detect the learning ability of rats [27]. An escape platform (diameter $10 \mathrm{~cm}$ ) was placed in the first quadrant (the target quadrant) of four quadrants divided by software, and was submerged $1-2 \mathrm{~cm}$ below the surface. Rats were put into the maze at the midpoint of the maze margin facing the wall. Each rat was trained four times a day. Memory tests were conducted by probe trial after withdrawing escape platform on the sixth day. Each animal was tested twice and limited for $120 \mathrm{~s}$ in each trial. The vision and motor function of animals were checked by a visual platform test, in which the escape platform was elevated $2 \mathrm{~cm}$ above the water and each animal was tested twice [28].

\subsection{In vivo hippocampal LTP recording}

Hippocampal LTP is an important indicator of neuronal plasticity. The LTP recording was performed in vivo with the same animal after undergoing behavioral tests. The rats were anesthetized with urethane ( $1.3 \mathrm{~g} / \mathrm{kg}$, i.p.), and a small hole ( $2.5 \mathrm{~mm}$ diameter) was drilled on the skull for inserting a pair of bounded stimulation/recording electrodes. The body temperature of rats was maintained at $37 \pm 0.5{ }^{\circ} \mathrm{C}$ with a heating pad throughout experimental period. The tip of concentric bipolar stimulating electrode (FHC, Inc., USA) was driven downward by a micromanipulator to the Schaffer collateral/commissural pathway with the following coordinates (using the bregma as a reference point): AP, $-4.2 \mathrm{~mm}$; ML, $-3.8 \mathrm{~mm}$ [29]. The tip position of recording electrode is the stratum radiatum in the hippocampus CA1 region with the following coordinates: AP, $-3.4 \mathrm{~mm}$; ML, $-2.5 \mathrm{~mm}$. The final vertical positions of the electrodes in the hippocampus were determined by electrophysiological criterion, an obvious negative deflecting field excitatory postsynaptic potential (fEPSP) evoked by a stimulus from stimulation isolator (Master-9, AMPI, Israel). The basal fEPSPs were evoked by continuous single electric stimulus (intensity: elicited $50 \%$ of maximal response; frequency: $0.033 \mathrm{~Hz}$ ) and recorded for $30 \mathrm{~min}$. LTP of fEPSPs was induced by high-frequency $(200 \mathrm{~Hz})$ stimulation (HFS) containing 20 pulses. $A>30 \%$ increase in the amplitude of fEPSP after HFS was considered successful induction of LTP. After that, fEPSPs were recorded at a frequency of $0.033 \mathrm{~Hz}$ for at least $60 \mathrm{~min}$. To determine whether presynaptic mechanisms were involved, paired pulse 
facilitation (PPF) was also examined with paired pulses after recording basal fEPSPs. All signals from recording electrode were sent to a signal acquisition and processing system (Chengdu Instruments Inc., China) for off-line analysis.

\subsection{Western blotting}

After electrophysiological experiment, rats were sacrificed under anesthesia. Bilateral hippocampi were removed and homogenized by ultrasonic crusher under cold condition. Homogenates were centrifuged at $14000 \mathrm{rmp}$ for $10 \mathrm{~min}$ at $4{ }^{\circ} \mathrm{C}$. The concentration of total protein in the supernatant was determined by Micro bicinchoninic acid (BCA) assay kit (Boster Co. Ltd. China). Protein samples ( $40 \mu \mathrm{g}$ ) were separated by electrophoresis on $12 \%$ SDS-polyacrylamide gels. After electrophoresis, proteins were transferred to PVDF membrane. Membranes were incubated with $5 \%$ bovine serum albumin (BSA) at $4{ }^{\circ} \mathrm{C}$ conditions for $2 \mathrm{~h}$ in order to block nonspecific binding. Then, membranes were incubated with first antibody include anti-p-AKT antibody (Ser473, 1:1000), and anti-AKT antibody $(1: 1000)$ at $4{ }^{\circ} \mathrm{C}$ for $12 \mathrm{~h}$. Thereafter, membranes were rinsed three times every five minutes by Tris-Buffered Saline with $0.05 \%$ Tween 20 (TBST), then incubated with secondary antibody (1:50,000, ZSGB-BIO, Inc. China) at room temperature for $2 \mathrm{~h}$. Afterwards, membranes were again rinsed three times with the same conditions as previously in TBST. The immunocomplexes were visualized by enhanced chemiluminescence using the ECL kit (Beyotime, Inc. China) according to manufacturer instructions. The signals of the membranes were scanned with ChemiDoc XRS + system and quantified with image lab 5.2 software (Bio-Rad, Inc. US). $\beta$-actin was used as an internal control for sample loading. AKT and p-AKT were normalized to their corresponding $\beta$-actin value.

\subsection{Data analysis}

All data were expressed as means \pm standard errors (SEM) and analyzed by statistical software SPSS13.0. Data of MWM in training days were calculated and compared with three-way ANOVA. Other data of behavioral experiments were calculated with two-way ANOVA. The data from electrophysiological and western blotting experiments were calculated by using two-way classification ANOVA. Statistical significance was assigned as $\mathrm{P}<0.05$.

\section{Results}

3.1. Intranasal administration of davunetide protected against $A \beta-42$ induced impairment of spatial learning and memory in rats

The spatial learning ability of rats was assessed by performing a consecutive 5-day underwater platform test in the MWM. As shown in the Fig. $1 \mathrm{~A}$, the mean escape latency of rats to find underwater platform gradually decreased in each group during 5 training days $\left(F_{(4,160)}=\right.$ $200.433, \mathrm{P}<0.001)$. Significant main effects existed in group saline vs davunetide treatment $\left[F_{(1,40)}=15.145, \mathrm{P}<0.01\right]$ and in group vehicle vs $A \beta-42$ injection $\left[F_{(1,40)}=12.115, \mathrm{P}<0.01\right]$ on escape latency. There was a significant interaction between $A \beta-42$ injection and davunetide treatment $\left[F_{(1,40)}=11.556, \mathrm{P}<0.01\right]$. Tukey's post hoc test showed that the escape latencies of rats in vehicle $+A \beta-42$ group were significantly increased on the training day 3 (46.43 $\pm 3.76 \mathrm{~s}$, $\mathrm{P}<0.01)$, day $4(44.34 \pm 3.54 \mathrm{~s}, \mathrm{P}<0.01)$ and day $5(39.94 \pm 3.87 \mathrm{~s}$, $\mathrm{P}<0.001)$ compared to that in vehicle + saline group on the training day $3(31.44 \pm 2.84 \mathrm{~s})$, day $4(24.02 \pm 3.40 \mathrm{~s})$, and day 5 (22.54 \pm $1.85 \mathrm{~s}$ ), indicating that $A \beta 1-42$ significantly damaged the spatial learning ability of rats. Importantly, davunetide treatment significantly decreased the escape latency of rats, being $(35.46 \pm 3.06 \mathrm{~s}, \mathrm{P}<0.01)$, $(27.72 \pm 4.21 \mathrm{~s}, \mathrm{P}<0.01),(25.27 \pm 3.86 \mathrm{~s}, \mathrm{P}<0.01)$, on day $3-5$, respectively, significantly lower than that in vehicle $+A \beta-42$ group. Typical swimming traces of all the four group animals on 4th training day were shown in Fig. 1E.

The spatial memory of rats was assessed by two times of prob. trial. In the test, the platform was removed, and each animal was permitted to swim for $120 \mathrm{~s}$. We found that there were significant main effects of davunetide treatment and $A \beta 1-42$ injection [davunetide treatment $\left[F_{(1,40)}=2.663, \mathrm{P}<0.05\right] ; \mathrm{A} \beta 1-42$ injection $\left[F_{(1,40)}=6.326, \mathrm{P}<0.01\right]$ and interaction effects between $A \beta 1-42$ injection and davunetide treatment $\left[F_{(1,40)}=7.896, \mathrm{P}<0.01\right]$. As shown in the Fig. $1 \mathrm{~B}$, the following Tukey's post hoc test showed that the significant decrease in the percentage of swimming time of rats in veilcle $+A \beta 1-42$ group (17.9 \pm $2.2 \%, \mathrm{P}<0.01)$ compared with the veilcle + saline group $(40.1 \pm$ $1.6 \%)$ had an obvious recovery in Davunetide $+\mathrm{A} \beta 1-42$ group $(36.9 \pm 2.9 \%, \mathrm{P}<0.05)$, compared with that in the veilcle $+\mathrm{A} \beta 1-42$ group. Fig. $1 \mathrm{~F}$ showed the typical swimming traces of rats in the probe trials in different groups.

To exclude the impact of motor ability and vision on the above tests, a visible platform test was performed after prob tests. In the visible platform test, the average swimming speed and swimming latency of rats to find the visible platform were compared. The results (Fig. 1) showed that there is no significant difference $(\mathrm{P}>0.05)$ in swimming speed (Fig. 1C) and swimming time to visible platform (Fig. 1C), indicating that both $A \beta 1-42$ and davunetide did not affect the athletic ability and visual of animals.

\subsection{2. Davunetide reversed Aß1-42-induced suppression of hippocampal LTP}

After water maze test, we recorded in vivo hippocampal LTP in the CA1 region of the same animals to analyze the association between spatial memory and synaptic plasticity. After $30 \mathrm{~min}$ of stable baseline fEPSP recording, three strings of HFS $(200 \mathrm{~Hz})$ were delivered to Schaffer collateral, and fEPSPs potentiated in the CA1 region were continuously recorded for $60 \mathrm{~min}$. Fig. 2A showed the changes of fEPSPs slope before and after HFS in different groups. Two-way ANOVA indicated that both davunetide treatment group and $A \beta 1-42$ injection group had significant main effects on the induction of LTP at $1 \mathrm{~min}$ after HFS [avunetide treatments: $F_{(1,40)}=5.483, \mathrm{P}<0.05$; A $\beta 1-42$ injection $\left.F_{(1,40)}=22.164, \mathrm{P}<0.001\right]$. There was also a significant interaction effect between davunetide treatment group and $A \beta 1-42$ injection group $\left[F_{(1,40)}=7.833, \mathrm{P}<0.01\right]$. As shown in the Fig. 2B, Tukey's post-hoc test showed that $A \beta 1-42$ injection significantly suppressed the induction of LTP $(\mathrm{n}=10, \mathrm{P}<0.001)$, while davunetide treatments reversed the suppression ( $\mathrm{n}=10, \mathrm{P}<0.01$ ). At $60 \mathrm{~min}$ after HFS (Fig. $2 \mathrm{C}$ ), both davunetide treatment group and $A \beta 1-42$ injection group had significant main effects on the maintenance of LTP [davunetide treatments: $F_{(1,40)}=24.103, \mathrm{P}<0.001 ; \mathrm{A} \beta 1-42$ injection $F_{(1,40)}=34.260$, $\mathrm{P}<0.001]$. There was also a significant interaction effect between avunetide treatment group and $A \beta 1-42$ injection group $\left[F_{(1,40)}=\right.$ 19.957, $\mathrm{P}<0.001$ ] (Fig. 2B). Tukey's post hoc test showed that AB1-42 injection can significantly suppressed the maintenance of LTP $(\mathrm{n}=$ $10, P<0.001$ ), which was reversed by davunetide treatments $(n=10$, $\mathrm{P}<0.001)$.

Further, we recorded paired-pulse facilitation (PPF) to clarify whether presynaptic mechanism was involved in the effects of davunetide and A 1-42 on synaptic plasticity. The ratio of second fEPSP over first fEPSP was compared (Fig. 2D). Statistical results showed that no significant difference between the groups ( $n=10, P>0.05$ ), suggesting $A \beta 1-42$ injection and davunetide treatment did not affect presynaptic neurotransmitter release in the rat hippocampal CA1 region.

\subsection{Davunetide mostly restored the level of phospharylated AKT in rat hippocampus}

The PI3K/Akt pathway has attracted attention for its pivotal role in regulating cell survival, proliferation, synaptic plasticity and cognitive 
A

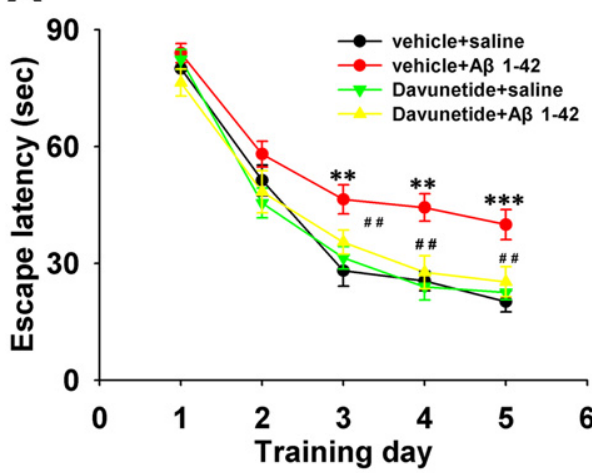

C

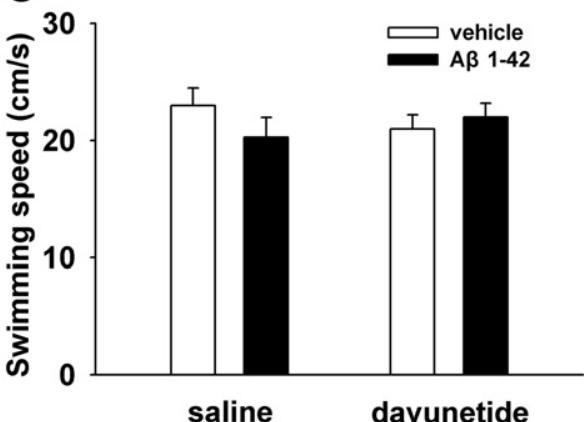

$\mathbf{E}$

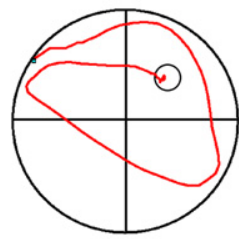

vehicle+saline

$\mathbf{F}$

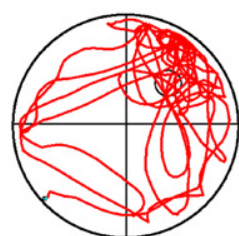

vehicle+saline

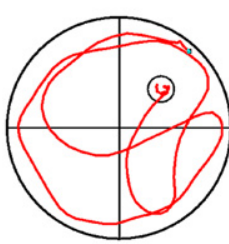

vehicle+Aß1-42

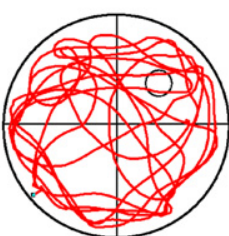

vehicle+A $\beta 1-42$

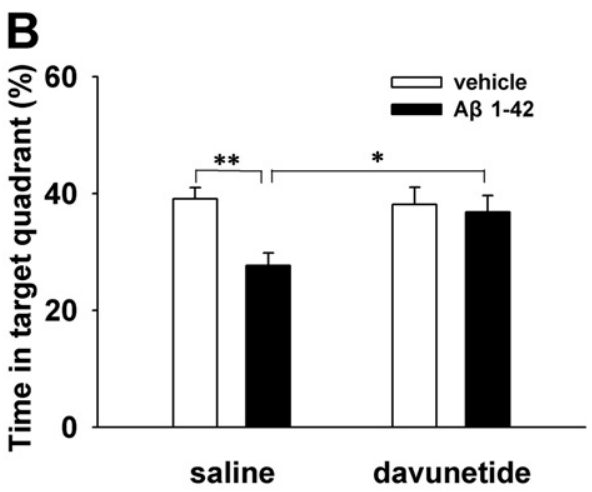

D
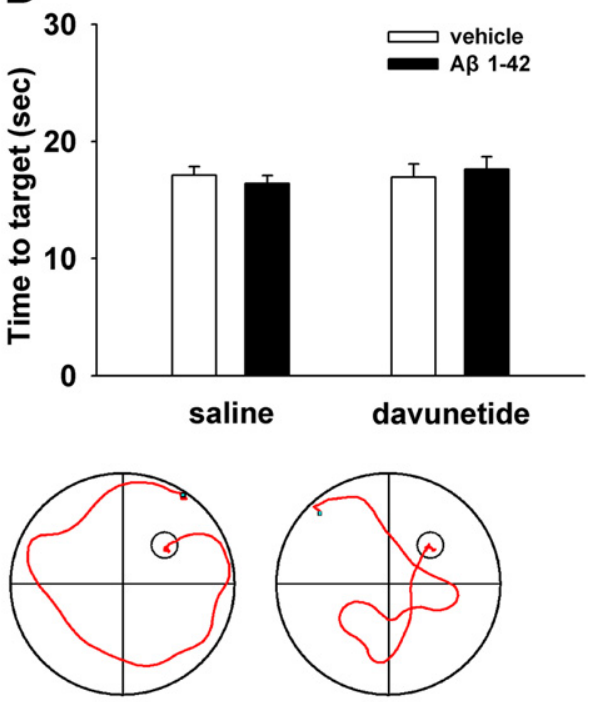

davunetide+saline
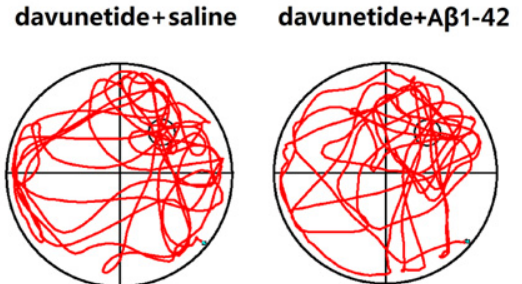

davunetide+saline

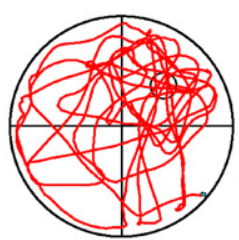

davunetide+A $\mathrm{A} 1-42$

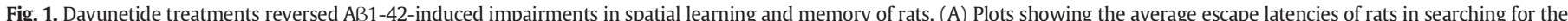

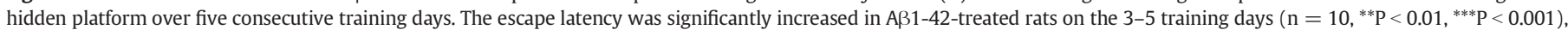

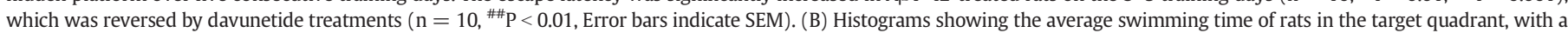

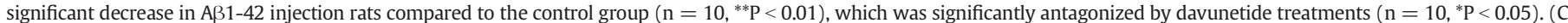

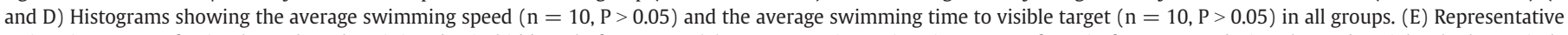

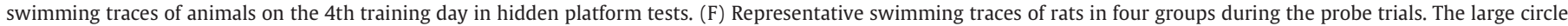
represents the Morris water maze pool and the small circle represents the platform.

performance [30,31]. To clarify whether the neuroprotection of davunetide in spatial memory and synaptic plasticity was associated with the regulation of PI3K/Akt signal pathway, we further examined the levels of total AKT and phosphorylated (Ser473) AKT (p-AKT) in the hippocampus. As shown in the Fig. 3 by Western blotting, Twoway ANOVA indicated that there are no main effects on the level of AKT (AKT/B-actin) between AB1-42 injection and davunetide treatment $\left[F_{(1,24)}=3.923, \mathrm{P}>0.05\right]$, suggesting no significant change in the total levels of Akt protein. However, A $1-42$ injection and davunetide treatment had significant main effects on the level of $\mathrm{p}$ AKT/AKT [Aß1-42 injection: $F_{(1,24)}=5.267, \mathrm{P}<0.05$; davunetide treatment: $\left.F_{(1,24)}=9.687, \mathrm{P}<0.01\right]$. Tukey's post hoc test showed that expression level of $\mathrm{p}-\mathrm{AKT}$ ( $\mathrm{p}$-AKT/AKT) in the hippocampus was decreased in $A \beta 1-42$-induced rats $(n=6, P<0.01)$, while davunetide treatment mostly and significantly rescued the level of $\mathrm{p}-\mathrm{AKT}$ ( $\mathrm{n}=6$, $\mathrm{P}<0.01$ ).

\section{Discussion}

Although the mechanism of AD is not fully understood, one of the popular proposals is the amyloid cascade hypothesis. $A \beta$ has been considered the initiator of subsequent events leading to the occurrence of $\mathrm{AD}$ [32]. It is well known that the spatial learning and memory function is closely dependent on the hippocampus in mammals, and any insults including neurotoxic $A \beta$ could impair the learning and memory behavior $[8,33]$. There are two major $A \beta$ isoforms, $A \beta 1-40$ and $A \beta 1-42$, in the brain of $A D$ patients [34]. Although $>90 \%$ of $A \beta$ isoforms is $A \beta 1-40, A \beta 1$ 42 oligomers are the most neurotoxic form [35]. In pathological 
A
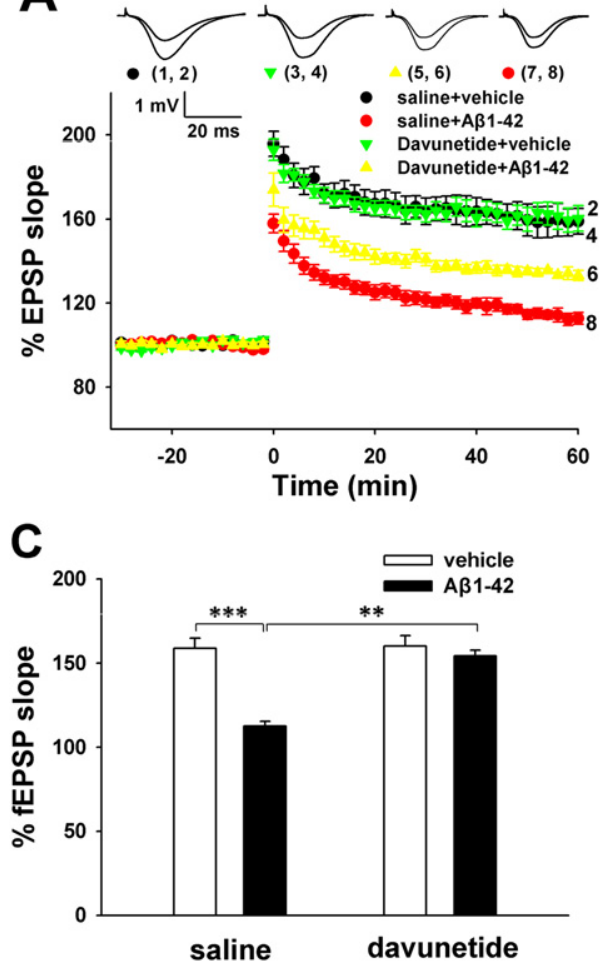

B
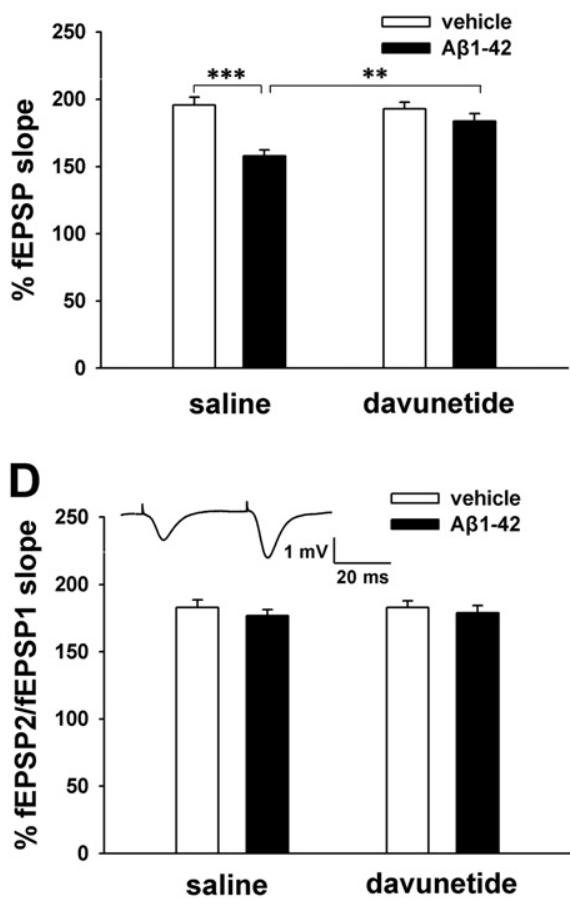

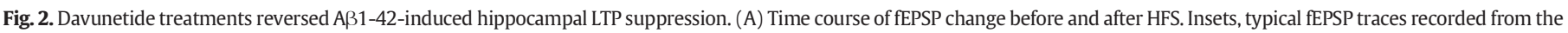

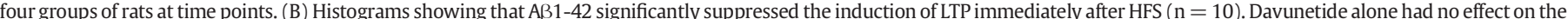

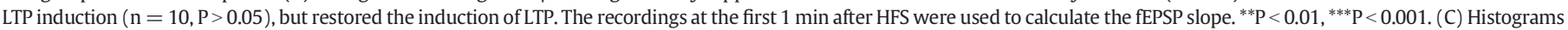

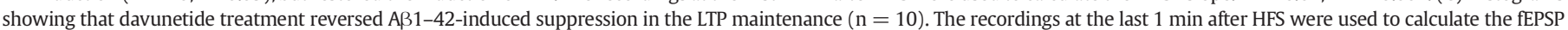

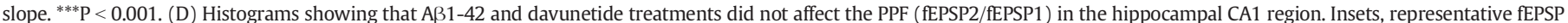
trace induced by paired pulses.

conditions, $A \beta$ molecules spontaneously gathered and deposited into soluble oligomers, fibrils and SPs, then caused oxidative damage and disturbance of kinase/phosphatase activity, eventually leading to neuronal death [36]. Therefore, aged A $\beta 1-42$ was used in the present study to mimic the neurodegenerative pathological processes of AD by directly injecting into the bilateral hippocampi of rats. As shown above, our results demonstrated that intrahippocampal injection of $A B 1-42$ not only impaired the spatial learning and memory of rats in MWM tests, but also suppressed LTP of fEPSPs in the hippocampal CA1 region on the same animals. These results are similar to our recent reports $[9,10]$.

In the central and peripheral nervous system, neurotrophic factors (NTFs) is capable of regulating neuronal survival and differentiation, and promoting the growth of new neurons and synapses $[37,38]$. These NTFs and their receptors, such as brain-derived neurotrophic

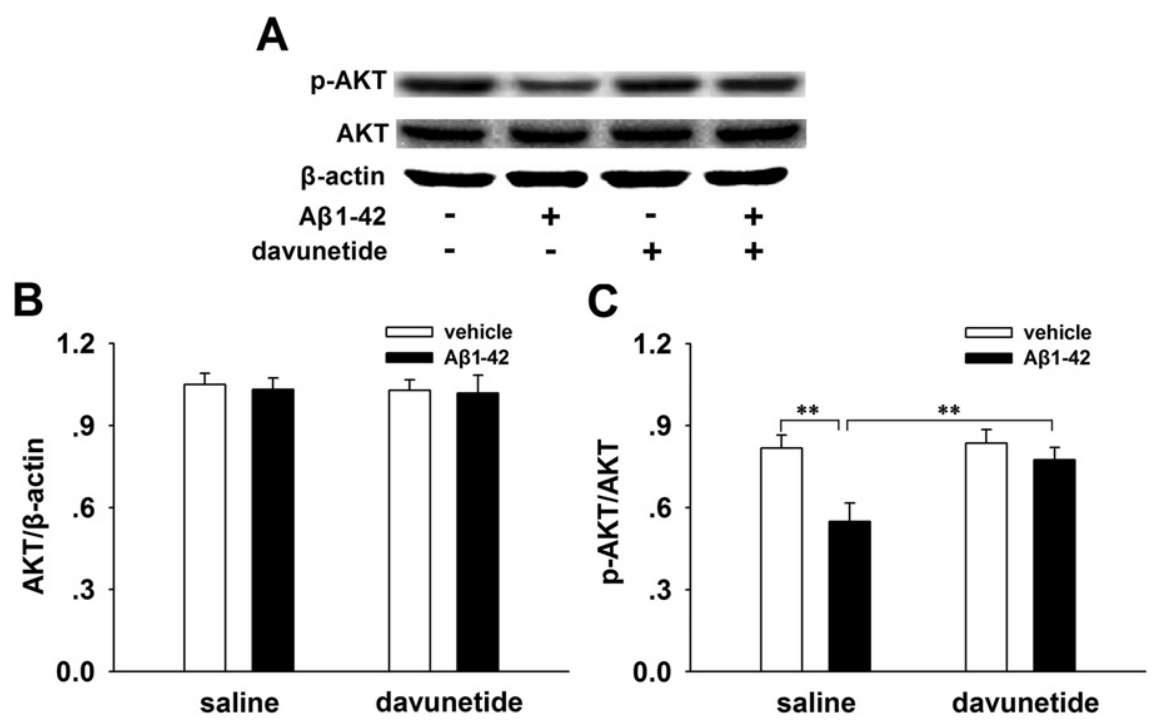

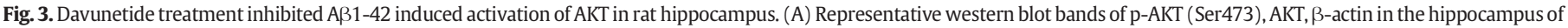

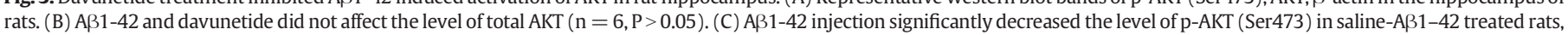
and this deficit was mostly restored by the davunetide treatment $\left(n=6,{ }^{* *} \mathrm{P}<0.05\right)$. Error bars indicate SEM. 
factor (BDNF) and TrkB receptors, are also active in the hippocampus, cortex, and basal forebrain areas vital to learning, memory and thinking [39]. Many studies have shown lowered levels of NTFs in the AD brain, suggesting that NTFs have a protective role against $A \beta$ toxicity and a very broad prospect in treating degenerative central nervous system diseases including AD $[40,41]$. However, NTFs have relatively larger molecular weight, in general, and are difficult to pass through the bloodbrain barrier (BBB). For example, nerve growth factor (NGF) is a $130 \mathrm{kDa}$ complex of three proteins and zinc ions, and its active region is beta-NGF, a single chain of two 118 amino acids joined by a noncovalent bond. BDNF consists of 119 amino acid residues and the molecular weight is $13.5 \mathrm{kDa}$. The molecular weight of ADNP is $124 \mathrm{kDa}$ [42], which greatly limits its clinical application [43]. In recent years, many short peptides were found to have strong effects of nutrition and protection. For instance, Humanin contains 14 amino acid residues [44,45]; ADNF-9 contains nine amino acid and davunetide (NAP) contains eight amino acid residues [46-48]. It is reported that davunetide protected neurons subjected to oxygen-glucose deprivation at very low concentrations by completely blocked cytochrome $c$ release [49]; davunetide could also cross the BBB after intranasal treatment and could be detected in rodents' brains by radio labeled peptides studies [50]; furtherly, intranasal administration davunetide could pass through olfactory nerve into the olfactory bulb, and finally to deep brain and cerebrospinal fluid, and play a role in nutrition and protect the central nervous system. Moreover, daily intranasal administration $1 \mu$ g davunetide significantly improved short-term spatial memory of rats impaired by cholinotoxin AF64A [50], and protected against developmental retardation in apolipoprotein E-deficient mice [51]. Another study found that davunetide reduce neonatal mouse brain cells apoptosis and excitotoxic brain damage [52]. Importantly, davunetide also prevented A $\beta$ aggregation in vitro experiments [19] and promoted the dissolution of $A \beta$ plaques in cortical cell and protected neurons against $A \beta$ neurotoxicity [19]. In the present in vivo study, the neuroprotective effect of davunetide against $A \beta 1-42$ was investigated by combination use of behavioral test and electrophysiological recording. In MWM test, we found that bilateral intrahippocampal injection of A $31-42$ significantly impaired spatial learning and memory of rats, with increased escape latency in hide platform test and decreased target quandrant swimming distance in probe trail; while three weeks intranasal administration of davunetide basically reversed these deficits induced by AB1-42. In hippocampal LTP recording, we found that davunetide did not affect the basic synaptic transmission, but protected against $A \beta 1-42$ induced suppression in hippocampal LTP. It is notable that our behavioral tests and electrophysiological recording were performed on the same animals. The consistency of the results from MWM and LTP tests helps us to best understand the close association between spatial memory and synaptic plasticity, and provides more convincing evidence that davunetide might be beneficial in attenuating AD-related deficits in learning and memory.

The PI3k/Akt pathway is an important intracellular signaling pathway in regulating cell survival, proliferation, and synaptic transmission [30]. The Akt kinase (also called protein kinase B) activity is induced following activation of PI3K in growth factor receptor mediated signaling cascades [30,53]. Upon receptor tyrosine kinase (RTK)-dependent activation by growth factors, PI3K complexes are recruited to the plasma membrane. In addition to lipid kinase activity and resulting PIP3 production, PI3Ks also exert serine/threonine protein kinase activity against protein substrates which leading to the phosphorylation and activation of Akt linked to membrane PIP3. Once activated, Akt promotes downstream biological responses. In neuronal cells, activated Akt regulates diverse targets that have been implicated in the regulation of protein translation and cell size, axonal outgrowth, apoptosis suppression and synaptic plasticity. Several lines of in vitro evidence using neuronal cell lines and dispersed primary neuronal cultures have demonstrated a requirement for Akt in the protection against trophic factor deprivation, oxidative stress and ischemic injury [54-56]. Other studies also have shown that Ras/MAPK and PI3k/Akt kinases mediated anti-apoptotic effect of BDNF against glutamate-induced neuronal death on excitotoxic brain damage model [57]. In support of a direct involvement of Akt in synaptic function, studies directed at working memory performance performed in Akt1 (-/ ) mice [58] found a strong correlation between Akt activity and cognitive performance. Additionally, this pathway has been found to be a necessary component in neural long term potentiation.

Interestingly, it has been shown that the PI3k/Akt pathway was realated to $A \beta$-induced memory impairment in drosophila [59]. Dysregulation of Akt activity was also observed in neurodegenerative diseases including AD [60]. Aß25-35 could reduce Akt activity on PC12 cells. Similarly, we found, in the present study that intrahippocampal injection of Aß1-42 oligomers did not affect the total Akt level, but induced a significant decrease in the level of pAKT, as well as impairments in spatial memory and synaptic plasticity. The PI3k/Akt pathway has also been implicated in the neuroprotective effects of some growth factors, such as BDNF and ADNP. Luckily, we found that chronic intranasal administration of davunetide not only prevented the pAKT decline, but also antagonized AB1-42 induced memory deficits and LTP suppression. Therefore, we deem that the improvements of spatial memory and synaptic plasticity by davunetide are probably involved in the activation of growth factor receptor mediated PI3k/Akt pathway. Thus, davunetide, or other activator of PI3k/Akt pathway, might be beneficial to the degenerative Alzheimer's disease. In fact, we have pleased to see that davunetide has been used in clinical trials in patients suffering from amnestic mild cognitive impairment, without significant side effect [61-63].

\section{Acknowledgments}

This work was supported by National Science Foundation of China (31271201, 31300968, 31471080), Graduate Students Outstanding Innovation Project Foundation of Shanxi Province (20143061) and 331 Early Career Researcher Grant of the Basic Medical School, Shanxi Medical University.

\section{References}

[1] A. Burns, S. Iliffe, Alzheimer's disease, BMJ 338 (2009) b158.

[2] R.J. O'Brien, P.C. Wong, Amyloid precursor protein processing and Alzheimer's disease, Annu. Rev. Neurosci. 34 (2011) 185-204.

[3] R. Vassar, Beta-secretase, APP and Abeta in Alzheimer's disease, Subcell. Biochem. 38 (2005) 79-103.

[4] J. Lauren, D.A. Gimbel, H.B. Nygaard, J.W. Gilbert, S.M. Strittmatter, Cellular prion protein mediates impairment of synaptic plasticity by amyloid-beta oligomers, Nature 457 (2009) 1128-1132.

[5] S.T. Ferreira, W.L. Klein, The Abeta oligomer hypothesis for synapse failure and memory loss in Alzheimer's disease, Neurobiol. Learn. Mem. 96 (2011) 529-543.

[6] A.C. Brorsson, B. Bolognesi, G.G. Tartaglia, S.L. Shammas, G. Favrin, I. Watson, et al., Intrinsic determinants of neurotoxic aggregate formation by the amyloid beta peptide, Biophys. J. 98 (2010) 1677-1684.

[7] G. Orban, K. Volgyi, G. Juhasz, B. Penke, K.A. Kekesi, J. Kardos, et al., Different electrophysiological actions of 24- and 72-hour aggregated amyloid-beta oligomers on hippocampal field population spike in both anesthetized and awake rats, Brain Res. 1354 (2010) 227-235.

[8] A. Stuchlik, S. Kubik, K. Vlcek, K. Vales, Spatial navigation: implications for animal models, drug development and human studies, Physiol. Res. 63 (Suppl. 1) (2014) S237-S249.

[9] J.Q. Tong, J. Zhang, M. Hao, J. Yang, Y.F. Han, X.J. Liu, et al., Leptin attenuates the detrimental effects of beta-amyloid on spatial memory and hippocampal later-phase long term potentiation in rats, Horm. Behav. 73 (2015) 125-130.

[10] X.H. Yue, X.J. Liu, M.N. Wu, J.Y. Chen, J.S. Qi, Amyloid beta protein suppresses hippocampal theta rhythm and induces behavioral disinhibition and spatial memory deficit in rats, Sheng Li Xue Bao 66 (2014) 97-106.

[11] S. Mandel, G. Rechavi, I. Gozes, Activity-dependent neuroprotective protein (ADNP) differentially interacts with chromatin to regulate genes essential for embryogenesis, Dev. Biol. 303 (2007) 814-824.

[12] R. Zamostiano, A. Pinhasov, E. Gelber, R.A. Steingart, E. Seroussi, E. Giladi, et al., Cloning and characterization of the human activity-dependent neuroprotective protein, J. Biol. Chem. 276 (2001) 708-714.

[13] R. Fernandez-Montesinos, M. Torres, D. Baglietto-Vargas, A. Gutierrez, I. Gozes, J. Vitorica, et al., Activity-dependent neuroprotective protein (ADNP) expression in 
the amyloid precursor protein/presenilin 1 mouse model of Alzheimer's disease, $J$ Mol. Neurosci. 41 (2010) 114-120.

[14] A. Malishkevich, G.A. Marshall, A.P. Schultz, R.A. Sperling, J. Aharon-Peretz, I. Gozes, Blood-borne Activity-Dependent Neuroprotective Protein (ADNP) is correlated with premorbid intelligence, clinical stage, and Alzheimer's disease biomarkers, J. Alzheimers Dis. 50 (2016) 249-260

[15] I. Divinski, L. Mittelman, I. Gozes, A femtomolar acting octapeptide interacts with tubulin and protects astrocytes against zinc intoxication, J. Biol. Chem. 279 (2004) 28531-28538.

[16] V.L. Smith-Swintosky, I. Gozes, D.E. Brenneman, M.R. D'Andrea, C.R. Plata-Salaman, Activity-dependent neurotrophic factor-9 and NAP promote neurite outgrowth in rat hippocampal and cortical cultures, J. Mol. Neurosci. 25 (2005) 225-238.

[17] L. Visochek, R.A. Steingart, I. Vulih-Shultzman, R. Klein, E. Priel, I. Gozes, et al., PolyADP-ribosylation is involved in neurotrophic activity, J. Neurosci. 25 (2005) $7420-7428$.

[18] Y. Ivashko-Pachima, C.L. Sayas, A. Malishkevich, I. Gozes, ADNP/NAP dramatically increase microtubule end-binding protein-Tau interaction: a novel avenue for protec tion against tauopathy, Mol. Psychiatry (2017, Jan 24) http://dx.doi.org/10.1038/ mp. 2016.255 (Epub ahead of print).

[19] O. Ashur-Fabian, Y. Segal-Ruder, E. Skutelsky, D.E. Brenneman, R.A. Steingart, E. Giladi, et al., The neuroprotective peptide NAP inhibits the aggregation of the beta-amyloid peptide, Peptides 24 (2003) 1413-1423.

[20] G.H. Doherty, D. Beccano-Kelly, S.D. Yan, F.J. Gunn-Moore, J. Harvey, Leptin prevents hippocampal synaptic disruption and neuronal cell death induced by amyloid beta, Neurobiol. Aging 34 (2013) 226-237.

[21] W.B. Stine, L. Jungbauer, C. Yu, M.J. LaDu, Preparing synthetic Abeta in different aggregation states, Methods Mol. Biol. 670 (2011) 13-32.

[22] J.K. Ryu, J.G. McLarnon, Thalidomide inhibition of perturbed vasculature and glialderived tumor necrosis factor-alpha in an animal model of inflamed Alzheimer's disease brain, Neurobiol. Dis. 29 (2008) 254-266.

[23] H.Y. Cai, C. Holscher, X.H. Yue, S.X. Zhang, X.H. Wang, F. Qiao, et al., Lixisenatide rescues spatial memory and synaptic plasticity from amyloid beta protein-induced impairments in rats, Neuroscience 277 (2014) 6-13.

[24] R.N. Alcalay, E. Giladi, C.G. Pick, I. Gozes, Intranasal administration of NAP, a neuroprotective peptide, decreases anxiety-like behavior in aging mice in the elevated plus maze, Neurosci. Lett. 361 (2004) 128-131.

[25] I. Magen, R. Ostritsky, F. Richter, C. Zhu, S.M. Fleming, V. Lemesre, et al., Intranasal NAP (davunetide) decreases tau hyperphosphorylation and moderately improves behavioral deficits in mice overexpressing alpha-synuclein, Pharmacol. Res. Perspect. 2 (2014) e00065.

[26] C.V. Vorhees, M.T. Williams, Morris water maze: procedures for assessing spatia and related forms of learning and memory, Nat. Protoc. 1 (2006) 848-858.

[27] R. D'Hooge, P.P. De Deyn, Applications of the Morris water maze in the study of learning and memory, Brain Res. Brain Res. Rev. 36 (2001) 60-90.

[28] X.H. Wang, L. Li, C. Holscher, Y.F. Pan, X.R. Chen, J.S. Qi, Val8-glucagon-like peptide-1 protects against Abeta1-40-induced impairment of hippocampal late-phase longterm potentiation and spatial learning in rats, Neuroscience 170 (2010) 1239-1248.

[29] W.N. Han, C. Holscher, L. Yuan, W. Yang, X.H. Wang, M.N. Wu, et al., Liraglutide protects against amyloid-beta protein-induced impairment of spatial learning and memory in rats, Neurobiol. Aging 34 (2013) 576-588.

[30] T.F. Franke, PI3K/Akt: getting it right matters, Oncogene 27 (2008) 6473-6488.

[31] H.Y. Tan, K.K. Nicodemus, Q. Chen, Z. Li, J.K. Brooke, R. Honea, et al., Genetic variation in AKT1 is linked to dopamine-associated prefrontal cortical structure and function in humans, J. Clin. Invest. 118 (2008) 2200-2208.

[32] J. Hardy, D.J. Selkoe, The amyloid hypothesis of Alzheimer's disease: progress and problems on the road to therapeutics, Science 297 (2002) 353-356.

[33] E.I. Moser, E. Kropff, M.B. Moser, Place cells, grid cells, and the brain's spatial representation system, Annu. Rev. Neurosci. 31 (2008) 69-89.

[34] J. Kim, L. Onstead, S. Randle, R. Price, L. Smithson, C. Zwizinski, et al., Abeta40 inhibits amyloid deposition in vivo, J. Neurosci. 27 (2007) 627-633.

[35] D.M. Walsh, I. Klyubin, J.V. Fadeeva, W.K. Cullen, R. Anwyl, M.S. Wolfe, et al., Naturally secreted oligomers of amyloid beta protein potently inhibit hippocampal long-term potentiation in vivo, Nature 416 (2002) 535-539.

[36] E.S. Musiek, D.M. Holtzman, Three dimensions of the amyloid hypothesis: time, space and 'wingmen', Nat. Neurosci. 18 (2015) 800-806.

[37] A. Acheson, J.C. Conover, J.P. Fandl, T.M. DeChiara, M. Russell, A. Thadani, et al., A BDNF autocrine loop in adult sensory neurons prevents cell death, Nature 374 (1995) 450-453.

[38] E.J. Huang, L.F. Reichardt, Neurotrophins: roles in neuronal development and function, Annu. Rev. Neurosci. 24 (2001) 677-736.

[39] K. Yamada, T. Nabeshima, Brain-derived neurotrophic factor/TrkB signaling in memory processes, J. Pharmacol. Sci. 91 (2003) 267-270.
[40] M. Wirenfeldt, A.A. Babcock, R. Ladeby, K.L. Lambertsen, F. Dagnaes-Hansen, R.G. Leslie, et al., Reactive microgliosis engages distinct responses by microglial subpopulations after minor central nervous system injury, J. Neurosci. Res. 82 (2005) 507-514.

[41] A. Ducray, S. Kipfer, A.W. Huber, R.H. Andres, R.W. Seiler, U. Schlattner, et al., Creatine and neurotrophin- $4 / 5$ promote survival of nitric oxide synthase-expressing interneurons in striatal cultures, Neurosci. Lett. 395 (2006) 57-62.

[42] I Vulih-Shultzman, A. Pinhasov, S. Mandel, N. Grigoriadis, O. Touloumi, Z. Pittel, et al., Activity-dependent neuroprotective protein snippet NAP reduces tau hyperphosphorylation and enhances learning in a novel transgenic mouse model, J. Pharmacol. Exp. Ther. 323 (2007) 438-449.

[43] D.X. Qin, X.L. Zou, W. Luo, W. Zhang, H.T. Zhang, X.L. Li, et al., Expression of some neurotrophins in the spinal motoneurons after cord hemisection in adult rats, Neurosci. Lett. 410 (2006) 222-227.

[44] L. Yuan, X.J. Liu, W.N. Han, Q.S. Li, Z.J. Wang, M.N. Wu, et al., [Gly14]-Humanin Protects Against Amyloid beta Peptide-Induced Impairment of Spatial Learning and Memory in Rats, Neurosci. Bull. 32 (2016) 374-382

[45] F. Guo, W. Jing, C.G. Ma, M.N. Wu, J.F. Zhang, X.Y. Li, et al., [Gly(14)]-humanin rescues long-term potentiation from amyloid beta protein-induced impairment in the rat hippocampal CA1 region in vivo, Synapse 64 (2010) 83-91.

[46] L. Beni-Adani, I. Gozes, Y. Cohen, Y. Assaf, R.A. Steingart, D.E. Brenneman, et al., A peptide derived from activity-dependent neuroprotective protein (ADNP) ameliorates injury response in closed head injury in mice, J. Pharmacol. Exp. Ther. 296 (2001) 57-63.

[47] R.R. Leker, A. Teichner, N. Grigoriadis, H. Ovadia, D.E. Brenneman, M. Fridkin, et al., NAP, a femtomolar-acting peptide, protects the brain against ischemic injury by reducing apoptotic death, Stroke 33 (2002) 1085-1092.

[48] M. Bassan, R. Zamostiano, A. Davidson, A. Pinhasov, E. Giladi, O. Perl, et al., Complete sequence of a novel protein containing a femtomolar-activity-dependent neuroprotective peptide, J. Neurochem. 72 (1999) 1283-1293.

[49] I. Zemlyak, R. Sapolsky, I. Gozes, NAP protects against cytochrome c release: inhibition of the initiation of apoptosis, Eur. J. Pharmacol. 618 (2009) 9-14.

[50] I. Gozes, E. Giladi, A. Pinhasov, A. Bardea, D.E. Brenneman, Activity-dependent neurotrophic factor: intranasal administration of femtomolar-acting peptides improve performance in a water maze, J. Pharmacol. Exp. Ther. 293 (2000) 1091-1098.

[51] I. Gozes, M. Bachar, A. Bardea, A. Davidson, S. Rubinraut, M. Fridkin, et al., Protection against developmental retardation in apolipoprotein E-deficient mice by a fatty neuropeptide: implications for early treatment of Alzheimer's disease, J. Neurobiol. 33 (1997) 329-342.

[52] P. Sokolowska, S. Passemard, A. Mok, L. Schwendimann, I. Gozes, P. Gressens, Neuroprotective effects of NAP against excitotoxic brain damage in the newborn mice: implications for cerebral palsy, Neuroscience 173 (2011) 156-168.

[53] B.M. Burgering, P.J. Coffer, Protein kinase B (c-Akt) in phosphatidylinositol-3-OH kinase signal transduction, Nature 376 (1995) 599-602.

[54] H. Dudek, S.R. Datta, T.F. Franke, M.J. Birnbaum, R. Yao, G.M. Cooper, et al., Regulation of neuronal survival by the serine-threonine protein kinase Akt, Science 275 (1997) 661-665.

[55] M. Salinas, D. Martin, A. Alvarez, A. Cuadrado, Akt1/PKBalpha protects PC12 cells against the parkinsonism-inducing neurotoxin 1-methyl-4-phenylpyridinium and reduces the levels of oxygen-free radicals, Mol. Cell. Neurosci. 17 (2001) 67-77.

[56] N. Noshita, A. Lewen, T. Sugawara, P.H. Chan, Akt phosphorylation and neuronal survival after traumatic brain injury in mice, Neurobiol. Dis. 9 (2002) 294-304.

[57] R.D. Almeida, B.J. Manadas, C.V. Melo, J.R. Gomes, C.S. Mendes, M.M. Graos, et al., Neuroprotection by BDNF against glutamate-induced apoptotic cell death is mediated by ERK and PI3-kinase pathways, Cell Death Differ. 12 (2005) 1329-1343.

[58] W.S. Lai, B. Xu, K.G. Westphal, M. Paterlini, B. Olivier, P. Pavlidis, et al., Akt1 deficiency affects neuronal morphology and predisposes to abnormalities in prefrontal cortex functioning, Proc. Natl. Acad. Sci. U. S. A. 103 (2006) 16906-16911.

[59] H.C. Chiang, L. Wang, Z. Xie, A. Yau, Y. Zhong, PI3 kinase signaling is involved in Abeta-induced memory loss in Drosophila, Proc. Natl. Acad. Sci. U. S. A. 107 (2010) 7060-7065.

[60] J. Ryder, Y. Su, B. Ni, Akt/GSK3beta serine/threonine kinases: evidence for a signalling pathway mediated by familial Alzheimer's disease mutations, Cell. Signal. 16 (2004) 187-200.

[61] I. Gozes, A. Stewart, B. Morimoto, A. Fox, K. Sutherland, D. Schmeche, Addressing Alzheimer's disease tangles: from NAP to AL-108, Curr. Alzheimer Res. 6 (2009) 455-460.

[62] A.L. Boxer, A.E. Lang, M. Grossman, D.S. Knopman, B.L. Miller, L.S. Schneider, et al. Davunetide in patients with progressive supranuclear palsy: a randomised, double-blind, placebo-controlled phase 2/3 trial, Lancet Neurol. 13 (2014) 676-685.

[63] I. Magen, I. Gozes, Davunetide: peptide therapeutic in neurological disorders, Curr. Med. Chem. 21 (2014) 2591-2598. 